L. GAJEK (Lódź)

\title{
AN IMPROPER CRAMER-RAO LOWER BOUND
}

1. Introduction. This paper is concerned with the original form of the Cramer-Rao inequality. This is only one among many possible inequalities (see Section 3.2 of $[1]$ for a survey and references). Let $(\mathscr{X}, \mathscr{B}, \mu)$ be an atbitrary measure space with $\mu$ sigma-finite. Let $X$ be a random variable (r.v.) taking values in $\mathscr{X}$ with probability distribution $P_{\theta}(d x)=f_{\theta}(x) \mu(d x)$ for $x \in \mathscr{X}$ and $\theta \in \Theta$, where $\Theta$ is a known interval of the real line. In the sequel it is assumed that $\mathscr{X}$ is the $n$-dimensional Euclidean space $(n \geqslant 1)$ and $\mathscr{B}$ is the Borel field on $\mathscr{X}$.

Suppose that $\tau$ is a real-valued estimable function on $\Theta$, not identically constant, and $t(X)$ is an estimator of $\tau(\theta)$. Under certain regularity assumptions (see [3]) we have

$$
\mathrm{E}_{\theta}[t(X)-\tau(\theta)]^{2} \geqslant B^{2}(\theta)+\left[\tau^{\prime}(\theta)+B^{i}(\theta)\right]^{2} / \operatorname{Var}_{\theta} \frac{\partial}{\partial \theta} \log f_{\theta}(X),
$$

Where the right-hand side depends on the estimator $t$ through the bias $B(\theta)$ $=\mathrm{E}_{\theta}(t(X))-\tau(\theta)$. Clearly, for any $\theta_{1} \in \Theta$ and $\varepsilon>0$ there exists an estimator $t$ such that the risk is determined by

$$
R\left(t, \theta_{1}\right)=\mathrm{E}_{\theta_{1}}\left[t(X)-\tau\left(\theta_{1}\right)\right]^{2} h\left(\theta_{1}\right)<\varepsilon
$$

for a given weight function $h: \Theta \rightarrow(0, \infty)$. However, if $\Theta=(\underline{\theta}, \bar{\theta})$, then it is not a trivial problem to prove that for an arbitrary $\varepsilon>0$ either there exists an estimator the risk of which, for example in $\theta$, in the sense of the limit, is smaller than $\varepsilon$ or it is impossible. Theorem 1 shows that if the $\operatorname{limit}_{\boldsymbol{\theta} \rightarrow \boldsymbol{\theta}} \tau(\theta)$ exists and is equal to 0 or $\pm \infty$, then, under some additional assumptions, it is impossible. In Theorem 2 a non-trivial lower bound is given for any regular estimator. It should be emphasized that this bound does not depend on the estimator and is the best of all possible to be obtained by the Cramer-Rao inequality. In the sequel this bound is called the improper Cramer-Rao lower bound (shortly, ICRB) in $\underline{\theta}$ (or $\bar{\theta}$ ).

It is easy to see that ICRB can be useful in proving minimaxity. In fact, Example 3 from Section 4 shows that proofs are then very simple, even in the case of not exponentially distributed estimators. 
In general, ICRB is smaller than the trivial lower bound in $\underline{\theta}$ (or $\bar{\theta}$ ) corresponding to the unbiased version of the Cramer-Rao inequality. However, these two bounds are equal to each other in some situations, for example in the case of estimating the mean for the normal distribution; the equality

$$
\lim _{\theta \rightarrow \underline{\theta}} \tau^{2}(\theta) h(\theta)=\infty
$$

is a sufficient condition under which both bounds coincide at the point $\underline{\theta}$. Theorem 3 gives an admissible bound for the class of regular estimators and can be easily adopted for proving admissibility (see Theorem 5). It is related to the result of Ibragimov and Has'minskii [2] for unbiased estimators.

2. Preliminary lemmas. First we prove the following extension of the de L'Hôspital rule:

Lemma 1. Let $D=(a, b) \backslash\left\{x_{0}\right\}$, where $x_{0}$ is adherent to $(a, b)$, and assume that $f, g: D \rightarrow R$ are differentiable. If $g^{\prime}(x) \neq 0$ for every $x \in D$ and

then

$$
\lim _{x \rightarrow x_{0}} f(x)=\lim _{x \rightarrow x_{0}} g(x)=0,+\infty \text { or }-\infty,
$$

$$
\liminf _{x \rightarrow x_{0}} \frac{f^{\prime}(x)}{g^{\prime}(x)} \leqslant \liminf _{x \rightarrow x_{0}} \frac{f(x)}{g(x)} \leqslant \limsup _{x \rightarrow x_{0}} \frac{f(x)}{g(x)} \leqslant \limsup _{x \rightarrow x_{0}} \frac{f^{\prime}(x)}{g^{\prime}(x)} .
$$

Proof. Consider the case

$$
\lim _{x \rightarrow x_{0}} f(x)=\lim _{x \rightarrow x_{0}} g(x)=0 .
$$

Let us define $f\left(x_{0}\right)=g\left(x_{0}\right)=0$. Thus, by the Cauchy theorem, for any $x \in D$ we have

$$
\frac{f(x)}{g(x)}=\frac{f(x)-f\left(x_{0}\right)}{g(x)-g\left(x_{0}\right)}=\frac{f^{\prime}(\xi)}{g^{\prime}(\xi)},
$$

where $\xi=x_{0}+\vartheta\left(x-x_{0}\right), 0<\vartheta<1$. Hence $\left|\xi-x_{0}\right|<\left|x-x_{0}\right|$ and for any $\delta>0$ we obtain

$$
\sup _{\left|x-x_{0}\right|<\delta} \frac{f^{\prime}(x)}{g^{\prime}(x)} \geqslant \sup _{\left|x-x_{0}\right|<\delta} \frac{f(x)}{g(x)} \geqslant \inf _{\left|x-x_{0}\right|<\delta} \frac{f(x)}{g(x)} \geqslant \inf _{\left|x-x_{0}\right|<\delta} \frac{f^{\prime}(x)}{g^{\prime}(x)} .
$$

Taking $\delta \rightarrow 0$, we complete the proof of this case.

Now, let

$$
\lim _{x \rightarrow x_{0}} f(x)=\lim _{x \rightarrow x_{0}} g(x)=\infty \quad \text { and } \quad \limsup _{x \rightarrow x_{0}} \frac{f^{\prime}(x)}{g^{\prime}(x)}=C .
$$


$1^{\circ}$ Suppose that $C=0$. We prove that

$$
\limsup _{x \rightarrow x_{0}} \frac{f(x)}{g(x)} \leqslant 0 \text {. }
$$

Let $x_{0} \in(a, b)$ (in the other case the proof is similar). Then for any $\varepsilon>0$ there exists a neighbourhood $U=\left(x_{0}-\Delta, x_{0}+\Delta\right)$ of $x_{0}$ such that for any $x \in U$, $x \neq x_{0}$, we have

$$
g(x)>0 \quad \text { and } \quad \frac{f^{\prime}(x)}{g^{\prime}(x)}<\frac{1}{2} \varepsilon
$$

By the identity

$$
\frac{f(x)}{g(x)}=\frac{f(\bar{x})}{g(x)}+\frac{f(x)-f(\bar{x})}{g(x)-g(\bar{x})}\left(1-\frac{g(\bar{x})}{g(x)}\right)
$$

and by the Cauchy theorem, for $x, \bar{x} \in U$ we obtain

$$
\frac{f(x)}{g(x)}=\frac{f(\bar{x})}{g(x)}+\frac{f^{\prime}(\xi)}{g^{\prime}(\xi)}\left(1-\frac{g(\bar{x})}{g(x)}\right)
$$

where $|x-\xi|<|x-\bar{x}|$. Since $\lim g(x)=\infty$, by (3) and (4) there exists $\delta>0$ such that $f(x) / g(x) \leqslant \varepsilon$ for all $x \in\left(x_{0}-\delta, x_{0}+\delta\right)$. Hence

$$
\limsup _{x \rightarrow x_{0}} f(x) / g(x) \leqslant 0 .
$$

$2^{\circ}$ Suppose that $|C|<\infty$. Let us define $f_{1}(x)=C g(x)$. Since

$$
\limsup _{x \rightarrow x_{0}} \frac{f_{1}^{\prime}(x)}{g^{\prime}(x)}=\limsup _{x \rightarrow x_{0}}\left(\frac{f^{\prime}(x)}{g^{\prime}(x)}-C\right)=0,
$$

from part $1^{\circ}$ it follows that

$$
\limsup _{x \rightarrow x_{\theta}} \frac{f_{1}(x)}{g(x)}=\limsup _{x \rightarrow x_{0}}\left(\frac{f(x)}{g(x)}-C\right) \leqslant 0 .
$$

This completes the proof of this part.

$3^{\circ}$ Suppose that $|C|=\infty$. In the case $C=\infty$ the assertion

$$
\limsup _{x \rightarrow x_{0}} \frac{f(x)}{g(x)} \leqslant \limsup _{x \rightarrow x_{0}} \frac{f^{\prime}(x)}{g^{\prime}(x)}
$$

is obvious. If $C=-\infty$, then

$$
\lim _{x \rightarrow x_{0}} \frac{f^{\prime}(x)}{g^{\prime}(x)}=-\infty
$$


but this contradicts the assumption

$$
\lim _{x \rightarrow x_{0}} f(x)=\lim _{x \rightarrow x_{0}} g(x)=\infty .
$$

Substituting $-f$ and $-g$ for $f$ and $g$, respectively, in $1^{\circ}-3^{\circ}$ we can prove the last inequality in (2) also for the case

$$
\lim _{x \rightarrow x_{0}} f(x)=\lim _{x \rightarrow x_{0}} g(x)=-\infty .
$$

It is easy to see that one can prove the first inequality in (2) analogously as the last one.

Remark 1. Under the assumptions of Lemma 1 suppose that $f^{\prime}(x) / g^{\prime}(x) \geqslant f(x) / g(x)$ on $D$. Then

$$
\liminf _{x \rightarrow x_{0}} f^{\prime}(x) / g^{\prime}(x)=\liminf _{x \rightarrow x_{0}} f(x) / g(x) .
$$

On the other hand, if $f(x) / g(x) \geqslant f^{\prime}(x) / g^{\prime}(x)$ on $D$, then

$$
\limsup _{x \rightarrow x_{0}} f(x) / g(x)=\limsup _{x \rightarrow x_{0}} f^{\prime}(x) / g^{\prime}(x) \text {. }
$$

Let 'us notice that by defining new functions $f_{1}(x)=f(1 / x)$ and $g_{1}(x)$ $=g(1 / x)$ one can extend Lemma 1 to the following

Lemma 2. Let $f, g:(a, \infty) \rightarrow R$ be differentiable functions such that $g^{\prime}(x) \neq 0$ for every $x>a$ and

$$
\lim _{x \rightarrow \infty} f(x)=\lim _{x \rightarrow \infty} g(x)=0,+\infty \text { or }-\infty
$$

Then

$$
\begin{aligned}
\underset{x \rightarrow \infty}{\liminf } \frac{f^{\prime}(x)}{g^{\prime}(x)} & \leqslant \liminf _{x \rightarrow \infty} \frac{f(x)}{g(x)} \leqslant \underset{x \rightarrow \infty}{\limsup } \frac{f(x)}{g(x)} \\
& \leqslant \limsup _{x \rightarrow \infty} \frac{f^{\prime}(x)}{g^{\prime}(x)} .
\end{aligned}
$$

The following simple example shows that in general the first and last inequalities in (2) and (5) cannot be changed to equality.

Example 1. Let $f(x)=x-x^{2} \sin 1 / x$ and $g(x)=x$ for $x \in(0,1)$. Then there exists

$$
\lim _{x \rightarrow 0} f(x) / g(x)=1
$$

but $\liminf _{x \rightarrow 0} f^{\prime}(x) / g^{\prime}(x)=0$ while

$$
\limsup _{x \rightarrow 0} \frac{f^{\prime}(x)}{g^{\prime}(x)}=2
$$


Now we investigate some limit properties of non-linear differential inequalities of the first order.

Let $a, b$ and $y$ be real functions defined on $J$, the interval of reals, such that $b(x)>a(x)>0$ for all $x \in J$, and $y$ is differentiable on $J$. Consider the following differential inequality:

$$
a(x) b(x)^{-1} \geqslant y^{2}(x) x^{-2}+a(x)[b(x)-a(x)]^{-1}\left[1+y^{\prime}(x)\right]^{2} .
$$

Lemma 3. Suppose that the real function $y \in C^{1}(J)$ satisfies (6) on the interval of reals $J$ and $x^{*}$ is a point adherent to $J$. If $x^{*} \in\{-\infty, 0, \infty\}$ and there exists

$$
\lim _{x \rightarrow x^{*}} a(x) / b(x),
$$

then there exists a sequence $\left\{x_{n}\right\}$ in $J$ such that $x_{n} \rightarrow x^{*}$ and

$$
\lim _{n \rightarrow \infty} y^{\prime}\left(x_{n}\right)=\lim _{n \rightarrow \infty} y\left(x_{n}\right) / x_{n}=-\lim _{n \rightarrow \infty} a\left(x_{n}\right) / b\left(x_{n}\right),
$$

(ii) $\lim _{n \rightarrow \infty} a\left(x_{n}\right) / b\left(x_{n}\right)=\lim _{n \rightarrow \infty}\left\{y^{2}\left(x_{n}\right) / x_{n}^{2}+a\left(x_{n}\right) \cdot\left[b\left(x_{n}\right)-a\left(x_{n}\right)\right]^{-1}\left[1+y^{\prime}\left(x_{n}\right)\right]^{2}\right\}$.

If, additionally, there exist

$$
\lim _{x \rightarrow x^{*}} b(x)=\infty \quad \text { and } \quad \lim _{x \rightarrow x^{*}} y(x)=0
$$

then

(iii)

$$
\lim _{n \rightarrow \infty} a\left(x_{n}\right)=\lim _{n \rightarrow \infty}\left\{y^{2}\left(x_{n}\right) / x_{n}^{2} b\left(x_{n}\right)+a\left(x_{n}\right)\left[1+y^{\prime}\left(x_{n}\right)\right]^{2}\right\} .
$$

Proof. One can find the idea of this proof in Example 8.4 of [4]. First we prove the assertions (i) and (ii).

I. Consider the case $x^{*}=0$. From (6) it follows that

$$
y^{2}(x) \leqslant x^{2} a(x) b(x)^{-1} \leqslant x^{2} .
$$

Thus

$$
\lim _{x \rightarrow x^{*}} y(x)=0
$$

and by Lemma 1 we have

$$
\begin{aligned}
\liminf _{x \rightarrow x^{*}} y^{\prime}(x) & \leqslant \liminf _{x \rightarrow x^{*}} y(x) / x \leqslant \underset{x \rightarrow x^{*}}{\limsup } y(x) / x \\
& \leqslant \limsup _{x \rightarrow x^{*}} y^{\prime}(x) .
\end{aligned}
$$

Suppose that

$$
\liminf _{x \rightarrow x^{*}} y^{\prime}(x)<\limsup _{x \rightarrow x^{*}} y^{\prime}(x) .
$$


Since $y^{\prime}$ is continuous on $J_{y}$, there exists a sequence $\left\{x_{n}\right\}$ such that $x_{n} \rightarrow x^{*}$ and

$$
y^{\prime}\left(x_{n}\right)=y\left(x_{n}\right) / x_{n} \quad \text { for } n=1,2, \ldots
$$

Let us notice that

$$
a / b \leqslant Z^{2}+a(b-a)^{-1}(1+Z)^{2}
$$

for any $b>a>0$ and for all $Z \in R$. Moreover, the equality in (9) holds if and only if $\bar{Z}=-a / b$. Thus from (6), (8) and (9) it follows that

$$
y^{\prime}\left(x_{n}\right)=y\left(x_{n}\right) / x_{n}=-a\left(x_{n}\right) / b\left(x_{n}\right)
$$

and the equality in (6) holds for the sequence $\left\{x_{n}\right\}$. Taking $n \rightarrow \infty$, we complete the proof of (i) and (ii) in this case.

Suppose that

$$
\liminf _{x \rightarrow x^{*}} y^{\prime}(x)=\underset{x \rightarrow x^{*}}{\limsup } y^{\prime}(x) \text {. }
$$

Then from (7) it follows that the limits of both sides of (8) exist for any sequence and

$$
\lim _{x \rightarrow x^{*}} y^{\prime}(x)=\lim _{x \rightarrow x^{*}} y(x) / x .
$$

Observe that (6) can be trànsformed as follows:

$$
\begin{aligned}
a(x) b(x)^{-1} \geqslant & y^{2}(x) x^{-2}+a(x)[b(x)-a(x)]^{-1}\left[1+y(x) x^{-1}\right]^{2}+ \\
& +a(x)[b(x)-a(x)]^{-1}\left\{\left[y^{\prime}(x)-y(x) x^{-1}\right]^{2}+\right. \\
& \left.+2\left[y^{\prime}(x)-y(x) x^{-1}\right]\left[1+y(x) x^{-1}\right]\right\} .
\end{aligned}
$$

From (10) and (11) it follows that

$$
\lim _{x \rightarrow x^{*}} a(x) b(x)^{-1} \geqslant \lim _{x \rightarrow x^{*}}\left\{y^{2}(x) x^{-2}+a(x)[b(x)-a(x)]^{-1}\left[1+y(x) x^{-1}\right]^{2}\right\} .
$$

On the other hand, (9) implies

$$
\lim _{x \rightarrow x^{*}} a(x) b(x)^{-1} \leqslant \lim _{x \rightarrow x^{*}}\left\{y^{2}(x) x^{-2}+a(x)[b(x)-a(x)]^{-1}\left[1+y(x) x^{-1}\right]^{2}\right\} .
$$

Thus the assertions (i) and (ii) follow from (12) and (13).

II. Let us consider the case $x^{*}=\infty$. From (6) we obtain

$$
-1-\sqrt{1-a(x) / b(x)} \leqslant y^{\prime}(x) \leqslant-1+\sqrt{1-a(x) / b(x)} .
$$

If

$$
\lim _{x \rightarrow x^{\prime}} a(x) / b(x)>0,
$$


then by (14) we have

$$
\lim _{x \rightarrow x^{4}} y(x)=-\infty
$$

and the proof is similar to that of the previous case. If

$$
\lim _{x \rightarrow x^{*}} a(x) / b(x)=0 \text {, }
$$

then

$$
\lim _{x \rightarrow x^{+}} y(x) / x=0
$$

by (6). It is easy to see that (14) implies $y^{\prime}(x) \leqslant 0$ as well. If

$$
\lim _{x \rightarrow x^{*}} y(x)=-\infty
$$

then the previous considerations may be applied. Otherwise, the function $y$ is non-increasing and bounded from below, and therefore it has a finite limit. However, this implies that

$$
\lim _{x \rightarrow x^{* *}} y^{\prime}(x)=0
$$

and the assertions hold.

III. The case $x^{*}=-\infty$ can be proved by analogy to case II.

Now we prove the assertion (iii). Let us notice that (6) is equivalent to

$$
a(x) \geqslant y^{2}(x) x^{-2} b(x)+\frac{b(x)}{b(x)-a(x)} a(x)\left[1+y^{\prime}(x)\right]^{2} .
$$

Since

$$
\lim _{x \rightarrow x^{*}} a(x)<\infty \quad \text { and } \quad \lim _{x \rightarrow x^{*}} b(x)=\infty,
$$

from (i) and the above inequality it follows that

$$
\lim _{n \rightarrow \infty} y\left(x_{n}\right) / x_{n}=\lim _{n \rightarrow \infty} y^{\prime}\left(x_{n}\right)=0
$$

and

$$
\lim _{n \rightarrow \gamma} a\left(x_{n}\right) \geqslant \limsup _{n \rightarrow \infty}\left[y^{2}\left(x_{n}\right) \dot{x}_{n}^{-2} b\left(x_{n}\right)\right]+\lim _{n \rightarrow \infty} a\left(x_{n}\right) .
$$

Hence there exists

$$
\lim _{n \rightarrow \infty}\left[y^{2}\left(x_{n}\right) x_{n}^{-2} b\left(x_{n}\right)\right]=0
$$

and (iii) holds. This completes the proof of Lemma 3 . 
Lemma 4. Suppose that the real function $y \in C^{1}(J)$ satisfies (6) on the interval $J=(\underline{x}, \bar{x})$. If $J=(-\infty, 0)$ or $(0, \infty)$ and there exist

$$
\lim _{x \rightarrow x} a(x) / b(x)=\lim _{x \rightarrow \bar{x}} a(x) / b(x),
$$

then

$$
y(x)=-C x \quad \text { for all } x \in J
$$

where

$$
C^{\prime}=\lim _{x \rightarrow x} a(x) / b(x)
$$

(ii) $a(x) / b(x)=y^{2}(x) x^{-2}+a(x)[b(x)-a(x)]^{-1}\left[1+y^{\prime}(x)\right]^{2}$ for all $x \in J$.

Proof. Let us notice that (6) implies $y^{\prime}(x) \leqslant y(x) x^{-1}$ for every $x \in J$, because otherwise by the inequalities

it follows from (6) that

$$
0 \geqslant y^{\prime}(x)>y(x) \dot{x}^{-1} \geqslant-\sqrt{a(x) / b(x)} \geqslant-1
$$

$$
\begin{aligned}
y^{2}(x) x^{-2} & +a(x)[b(x)-a(x)]^{-1}\left[1+y^{\prime}(x)\right]^{2} \\
& >y^{2}(x) x^{-2}+a(x)[b(x)-a(x)]^{-1}\left[1+y(x) x^{-1}\right]^{2}>a(x) / b(x),
\end{aligned}
$$

which contradicts (6). Therefore, $y(x) x^{-1}$ is a monotonic function, since

Assuming that

$$
\frac{d}{d x}\left[y(x) x^{-1}\right]=\left[y^{\prime}(x)-y(x) / x\right] x^{-1}
$$

$$
\lim _{x \rightarrow x} a(x) / b(x)=\lim _{x \rightarrow x} a(x) / b(x)=C
$$

and applying Lemma 3 we obtain

$$
\lim _{x \rightarrow x} y(x) / x=\lim _{x \rightarrow \bar{x}} y(x) / x=-C .
$$

Hence the function $y(x) x^{-1}$ is constant for $x \in(\underline{x}, \dot{\bar{x}})$ and $y(x)=-C x$. Then the assertion (ii) holds as well.

3. Improper Cramer-Rao lower bounds. Let $\lambda$ denote the Lebesgue measure on $\Theta$. The following regularity conditions insure the existence of the Cramer-Rao lower bound (1) (see [3]):

(a) $\Theta$ is an interval which may be finite or infinite;

(b) the set $M=\left\{(x, \theta) \in \not X \times \Theta:(\partial / \partial \theta) \log f_{\theta}(x)\right.$ exists\} belongs to $(. \not R \times: \not B)$ and on $M$ the function $(\partial / \partial \theta) \log f_{\theta}(x)$ is $(\mathscr{B} \times \not \mathscr{B})$-measurable;

(c) $0<\operatorname{Var}_{\theta}\left[(\partial / \partial \theta) \log f_{\theta}(X)\right]<\infty$ a.e. $[\lambda]$; a.e. $[$ l.]:

(d) $\int f_{\theta}(x) d \mu(x)$ is differentiable with respect to $\theta$ under the integral sign 
(e) $\mathrm{E}_{\theta}(t(X))=\int t(x) f_{\theta}(x) d \mu(x)$ is continuously differentiable under the integral sign a.e. [ $\lambda]$ and the statistic $t(x)$ is not equal to some constant a.e. $[\mu]$.

TheOREM 1. Suppose that the parameter space $\Theta$ contains a subset $\Theta^{*}$ on which $\tau$ is a diffeomorphism, the conditions (a)-(e) are satisfied and $\theta^{*}$ is a point adherent to $\Theta^{*}$ such that $\lim _{\theta \rightarrow \theta^{*}} \tau(\theta)$ exists and is equal to 0 or $\pm \infty$. If there exists a function $r(\theta)$ such that $0<r(\theta)<\tau^{2}(\theta) h(\theta)$ on $\Theta^{*}$ and the limits $\lim _{\theta \rightarrow \theta^{*}} \tau^{2}(\theta) h(\theta)$ and $\lim _{\theta \rightarrow \theta^{*}} r(\theta)<\infty$ exist, and

where

$$
\infty>\lim _{\theta \rightarrow \theta^{*}}\left\{\left[\tau^{2}(\theta) h(\theta) r(\theta)^{-1}-1\right]\left(\tau^{\prime}(\theta) / \tau(\theta)\right)^{2} I(\theta)^{-1}\right\}>1,
$$

$$
I(\theta)=\operatorname{Var}_{\theta}\left[\frac{\partial}{\partial \theta} \log f_{\theta}(X)\right]
$$

then there exists no regular (in the sense of condition (e)) estimator $t(X)$ such that

$$
\underset{\theta \rightarrow \theta}{\limsup } R(t, \theta)<\lim _{\theta \rightarrow \theta^{*}} r(\theta),
$$

Proof. Let us notice that if

$$
\lim _{\theta \rightarrow \theta} r(\theta)=0,
$$

then the assertion holds. Suppose that $\lim _{\theta \rightarrow \theta^{*}} r(\theta) \neq 0$ and there exists $t(X)$ such that

$$
\limsup _{\theta \rightarrow \theta^{* *}} R(t, \theta)<\lim _{\theta \rightarrow \theta^{*}} r(\theta) \text {. }
$$

Then there exist $\varepsilon_{0}>0$ and a set $\Theta_{0} \subseteq \Theta^{*}$ such that

$$
R(t, \theta) \leqslant r(\theta)-\varepsilon_{0} \quad \text { for every } \theta \in \Theta_{0},
$$

and $\theta^{*}$ is adherent to $\Theta_{0}$. Moreover, on the set $\Theta_{0}$ the Cramer-Rao inequality

$$
R(t, \theta) \geqslant B^{2}(\theta) h(\theta)+\left[\tau^{\prime}(\theta)+B^{\prime}(\theta)\right]^{2} h(\theta) I(\theta)^{-1}
$$

holds, where $B(\theta)=\mathrm{E}_{\theta}(t(X))-\tau(\theta)$ is the bias of $t$. From (15) it follows that there exists a set $\Theta_{1}$ such that $\theta^{*}$ is adherent to $\Theta_{1}$ and

$$
\left[\tau^{2}(\theta) h(\theta) r(\theta)^{-1}-1\right]\left(\tau^{\prime}(\theta) / \tau(\theta)\right)^{2} I(\theta)^{-1} \geqslant 1
$$

for every $\theta \in \Theta_{1}$. By (16) $(18)$, for every $\theta \in \Theta_{0} \cap \Theta_{1}$ the following inequality
holds: (19) $r(\theta)-\varepsilon_{0} \geqslant B^{2}(\theta) h(\theta)+r(\theta)\left[\tau^{2}(\theta) h(\theta)-r(\theta)\right]^{-1}\left(1+\frac{d B}{d \tau}\right)^{2} \tau^{2}(\theta) h(\theta)$. 
Dividing (19) by $\tau^{2}(\theta) h(\theta)$, we obtain

$$
\frac{r(\theta)-\varepsilon_{0}}{\tau^{2}(\theta) h(\theta)} \geqslant B^{2}(\theta) \tau(\theta)^{-2}+r(\theta)\left[\tau^{2}(\theta) h(\theta)-r(\theta)\right]^{-1}\left(1+\frac{d B}{d \tau}\right)^{2} .
$$

Thus

(21) $r(\theta)\left[\tau_{\ldots}^{2}(\theta) h(\theta)\right]^{-1} \geqslant B^{2}(\theta) \tau(\theta)^{-2}+r(\theta)\left[\tau^{2}(\theta) h(\theta)-r(\theta)\right]^{-1}\left(1+\frac{d B}{d \tau}\right)^{2}$

for every $\theta \in \Theta_{0} \cap \Theta_{1}$. Since $\tau$ is a diffeomorphism on $\Theta^{*} \supseteq \Theta_{0} \cap \Theta_{1}$, by Lemma 3 (ii) there exists a sequence $\left\{\theta_{n}\right\}$ such that $\theta_{n} \in \Theta_{0} \cap \Theta_{1}$ for all $n$, $\theta_{n} \rightarrow \theta^{*}(n \rightarrow \infty)$ and the equality in (21) holds in the sense of the limit of the sequence $\left\{\theta_{n}\right\}$. Observe that this contradicts $(20)$ in the case

$$
\lim _{\theta \rightarrow \theta^{*}} \tau^{2}(\theta) h(\theta)<\infty
$$

If

$$
\lim _{\theta \rightarrow \theta} \tau^{2}(\theta) h(\theta)=\infty
$$

then from (20) we obtain

$$
r(\theta)-\frac{\varepsilon_{0}}{2} \geqslant B^{2}(\theta) \tau(\theta)^{-2}\left(\tau^{2}(\theta) h(\theta)\right)+r(\theta) \frac{\tau^{2}(\theta) h(\theta)}{\tau^{2}(\theta) h(\theta)-r(\theta)}\left(1+\frac{d B}{d \tau}\right)^{2}
$$

and by Lemma 3 (iii) we have

$$
\begin{aligned}
\lim _{n \rightarrow \infty} r\left(\theta_{n}\right)= & \lim _{n \rightarrow \infty}\left\{B^{2}\left(\theta_{n}\right) \tau\left(\theta_{n}\right)^{-2}\left(\tau^{2}\left(\theta_{n}\right) h\left(\theta_{n}\right)\right)+\right. \\
& \left.+r\left(\theta_{n}\right)\left(1+\frac{d B}{d \tau}\left(\theta_{n}\right)\right)^{2}\right\}
\end{aligned}
$$

which leads to a contradiction, since

$$
\lim _{\theta \rightarrow \theta^{*}} r(\theta)<\infty .
$$

This completes the proof of Theorem 1.

Theorem 1 gives sufficient conditions under which the upper limits of risks of regular estimators are bounded from below at the endpoints of the parameter space: The best lower bound for this upper limit is given in the following

THEOREM 2. Suppose that the parameter space $\Theta$ contains a subset $\Theta^{*}$ on $^{n}$ which $\tau$ is a diffeomorphism, the conditions (a)-(e) are satisfied and $\theta^{*}$ is a point adherent to $\Theta^{*}$ such that $\lim _{\theta \rightarrow \theta^{*}} \tau(\theta)$ exists and is equal to 0 or $\pm \infty$. If there exist

$$
\lim _{\theta \rightarrow \theta^{*}} \tau^{2}(\theta) h(\theta)
$$


and

$$
\lim _{\theta \rightarrow \theta^{* 4}}\left\{\tau^{2}(\theta) h(\theta)\left[\left(\tau^{\prime}(\theta) / \tau(\theta)\right)^{-2} I(\theta)+1\right]^{-1}\right\}<\infty,
$$

then for every estimator $t(X)$ (regular in the sense of condition (e)) the following inequality holds:

$$
\limsup _{\theta \rightarrow \theta^{*}} R(t, \theta) \geqslant \lim _{\theta \rightarrow \theta^{*}} \tau^{2}(\theta) h(\theta)\left[\left(\tau^{\prime}(\theta) / \tau(\theta)\right)^{-2} I(\theta)+1\right]^{-1} .
$$

In the sequel the right-hand side of (22) is called the improper CramerRao lower bound (ICRB).

Proof. Consider the function

$$
r_{k}(\theta)=\tau^{2}(\theta) h(\theta)\left[k^{-1}\left(\tau^{\prime}(\theta) / \tau(\theta)\right)^{-2} I(\theta)+1\right]^{-1}
$$

for any $k(0<k<1)$. From the assumptions it follows that there exists $\lim _{\theta \rightarrow \theta^{*}} r_{k}(\theta)$. It is easy to check that

$$
\lim _{\theta \rightarrow \theta^{+}}\left\{\left[\tau^{2}(\theta) h(\theta) r_{k}(\theta)^{-1}-1\right]\left(\tau^{\prime}(\theta) / \tau(\theta)\right)^{2} I(\theta)^{-1}\right\}=k^{-1}>1 .
$$

Since $r_{k}(\theta)<\tau^{2}(\theta) h(\theta)$ for all $\theta$, the assumptions of Theorem 1 are satisfied, and therefore

$$
\limsup _{\theta \rightarrow \theta^{*}} R(t, \theta) \geqslant \lim _{\theta \rightarrow \theta^{*}} r_{k}(\theta)
$$

for any regular (in the sense of (e)) estimator $t(X)$ and for every $k(0<k$ $<1)$. Since

$$
\begin{aligned}
r_{k}(\theta) & =k \tau^{2}(\theta) h(\theta)\left[\left(\tau^{\prime}(\theta) / \tau(\theta)\right)^{-2} I(\theta)+k\right]^{-1} \\
& \geqslant k \tau^{2}(\theta) h(\theta)\left[\left(\tau^{\prime}(\theta) / \tau(\theta)\right)^{-2} I(\theta)+1\right]^{-1},
\end{aligned}
$$

from (23) and (24) it follows that

$$
\limsup _{\theta \rightarrow \theta^{\prime}} R(t, \theta) \geqslant \lim _{\theta \rightarrow \theta^{*}} \tau^{2}(\theta) h(\theta)\left[\left(\tau^{\prime}(\theta) / \tau(\theta)\right)^{-2} I(\theta)+1\right]^{-1} .
$$

This completes the proof.

Let us notice that

$$
\lim _{\theta \rightarrow \theta^{*}} \frac{\tau^{2}(\theta) h(\theta)}{\left(\tau^{\prime}(\theta) / \tau(\theta)\right)^{-2} I(\theta)} \geqslant \lim _{\theta \rightarrow \theta^{*}} \frac{\tau^{2}(\theta) h(\theta)}{\left(\tau^{\prime}(\theta) / \tau(\theta)\right)^{-2} I(\theta)+1},
$$

provided both limits exist, Clearly, the left-hand side of $(25)$ is the limit value of the unbiased Cramer-Rao lower bound. It is better (i.e. larger) but holds for a proper subset of all regular estimators only, while ICRB holds for the whole class. It is a surprising fact that these bounds differ from each other by the number 1 in the denominators. 
Suppose that both bounds are positive. Then the equality in (25) holds if and only if

$$
\lim _{\theta \rightarrow \theta^{*}} \tau^{2}(\theta) h(\theta)=\infty
$$

Example 1. Consider the r.v. $X$ with the gamma distribution

$$
f(x, \theta)=\theta^{s} / \Gamma(s) x^{s-1} \exp (-\theta x),
$$

where $f(x, \theta)$ is a density function of $X$ with respect to the Lebesgue measure and $x>0, \theta \in \Theta$. Suppose that $\Theta$ is of the form

(a) $\Theta=\left(0, \theta_{0}\right)$,

(b) $\Theta=\left(\theta_{0}, x\right)$,

and the parameter $\tau(\theta)=\theta^{-1}$ is estimated under the squared error loss with the weight function $h(\theta)=\theta^{2}$. Then in both cases (a) and (b) ICRB is equal to $(s+1)^{-1}$.

Example 2. Suppose that the r.v. $X$ is normally distributed $N(\theta, 1)$ and $\theta \in \Theta$. Let

(a) $\Theta=\left(-\infty, \theta_{0}\right)$,

(b) $\Theta=\left(\theta_{0}, \infty\right)$,

and let the parameter $\tau(\theta)=\theta$ be estimated under the squared error loss with the weight function $h(\theta)=1$. Then in both cases (a) and (b) ICRB is equal to 1 . Observe that this is the limit value of the unbiased Cramer-Rao lower bound as well.

Now we consider the case where $\Theta=(\theta, \theta)$ and where the limits of the function $\tau(\theta)$ at the endpoints $\underline{\theta}$ and $\bar{\theta}$ are equal to 0 or $\pm \infty$. Then Theorem 2 could be applied for both endpoints separately, but Lemma 4 enables us to obtain a more specified result.

THEOREM 3. Suppose that $\tau$ is a diffeomorphism on $\Theta=(\underline{\theta}, \bar{\theta})$, the conditions (a)-(e) are satisfied on $\Theta$, and $\tau(\Theta)=(-\infty, 0)$ or $\tau(\Theta)=(0, x)$. If there exist

$$
\lim _{\theta \rightarrow \underline{0}}\left[\left(\tau^{\prime}(\theta) / \tau(\theta)\right)^{-2} I(\theta)+1\right]^{-1}=\lim _{\theta \rightarrow \bar{\theta}}\left[\left(\tau^{\prime}(\theta) / \tau(\theta)\right)^{-2} I(\theta)+1\right]^{-1},
$$

then there exists no regular (in the sense of (e)) estimator $t(X)$ such that

$$
R(t, \theta) \leqslant \tau^{2}(\theta) h(\theta)\left[\left(\tau^{\prime}(\theta) / \tau(\theta)\right)^{-2} I(\theta)+1\right]^{-1}
$$

for every $\theta \in \Theta$ with the sharp inequality holding for some $\theta \in \Theta$.

Proof. Suppose that' there exists an estimator $t(X)$ such that (26) holds for every $\theta \in \Theta$ and the sharp inequality holds for some $\theta_{1} \in \Theta$. Hence, by the Cramer-Rao inequality, we have

$$
\tau^{2}(\theta) h(\theta)\left[\left(\tau^{\prime}(\theta) / \tau(\theta)\right)^{-2} I(\theta)+1\right]^{-1} \geqslant B^{2}(\theta) h(\theta)+\left[\tau^{\prime}(\theta)+B^{\prime}(\theta)\right]^{2} I^{-1}(\theta) h(\theta)
$$


Thus, after dividing both sides of this inequality by $\tau^{2}(\theta) h(\theta)$, we obtain (27)

$$
\begin{aligned}
{\left[\left(\tau^{\prime}(\theta) / \tau(\theta)\right)^{-2} I(\theta)+1\right.} & ]^{-1} \\
& \geqslant B^{2}(\theta) \tau^{-2}(\theta)+\left[\tau^{\prime}(\theta)+B^{\prime}(\theta)\right]^{2} I^{-1}(\theta) \tau^{-2}(\theta) .
\end{aligned}
$$

Since $\tau$ is a diffeomorphism on $\Theta$, from (27) we obtain

$$
\frac{a(\tau)}{b(\tau)} \geqslant B^{2}(\tau) \tau^{-2}+a(\tau)[b(\tau)-a(\tau)]^{-1}\left[1+\frac{d B(\tau)}{d \tau}\right]^{2},
$$

where $a(\tau) \equiv 1$ and $b(\tau(\theta))=\left(\tau^{\prime}(\theta) / \tau(\theta)\right)^{-2} I(\theta)+1$. By the assumptions there exist, and are equal to each other, both limits of $a(\tau) / b(\tau)$ at the endpoints of the interval $\tau(\Theta)$. Thus, by Lemma 4 , the equality holds in (28) for every $\tau \in \tau(\Theta)$ but this is a contradiction to (26) for $\tau_{1}=\tau\left(\theta_{1}\right)$.

Observe that now, comparing with Theorem 2, we are not interested in a reasonable bound for the risk at the endpoints of $\Theta$; therefore, the assumption on the limits of the expression

$$
\tau^{2}(\theta) h(\theta)\left[\left(\tau^{\prime}(\theta) / \tau(\theta)\right)^{-2} I(\theta)+1\right]^{-1}
$$

is omitted in Theorem 3. However, roughly speaking, the assumptions of Theorem 3 are stronger than other assumptions of Theorem 2 .

Remark 1. Theorem 3 concerns the case where $\tau$ is an increasing function. If $\tau$ is a decreasing function, then, after a change of variables $Q=$ $-\theta$, we can apply it in this case as well.

Let us consider the case $\tau(\Theta)=(-\infty, \infty)$. This case can be treated as a combination of two special cases of Theorem 3 and, under an additional assumption about the limit of $\left(\tau^{\prime}(0) / \tau(\theta)\right)^{-2} I(\theta)+1$ in 0 , the assertion holds. Ibragimov and Has'minskii [2] proved a similar result concerning the admissibility of unbiased estimators. We recall it in a convenient form.

THEOREM (Ibragimov and Has'minskii) If $\tau$ is a diffeomorphism on $\Theta$, $\tau(\Theta)=(-\infty, \infty)$, and

$$
\int_{\tau_{0}}^{\infty} I(\tau) d \tau=\infty \quad \text { and } \quad \int_{-\infty}^{\tau_{0}} I(\tau) d \tau=\infty,
$$

where

$$
I(\tau)=\mathrm{E}\left[\frac{\hat{\partial}}{\partial \tau} \log f(X, \tau)\right]^{2}, \quad \tau_{0} \in \tau(\Theta),
$$

then there exists no regular (in the sense of (e)) estimator $t(X)$ such that (30)

$$
R(t, \theta) \leqslant \tau^{2}(\theta) h(\theta)\left[\left(\tau^{\prime}(\theta) / \tau(\theta)\right)^{-2} I(\theta)\right]^{-1}
$$

for ecery $0 \in \Theta$ with the sharp inequality holding for some $\theta \in \Theta$. 
Clearly, the right-hand side of (30) is greater than the corresponding one of (26). Observe that (29) implies

$$
\left[\left(\tau^{\prime}(\theta) / \tau(\theta)\right)^{-2} I(\theta)\right]^{-1} \rightarrow 0 \quad \text { for } \tau(\theta) \rightarrow-\infty \text { and } \tau(\theta) \rightarrow \infty .
$$

This is an additional assumption, when compared with Theorem 3, which makes the assertion stronger. However, ICRB is the same as the corresponding bound for unbiased estimators.

4. Applications: The following result is an immediate consequence of Theorem 2 and the definition of a minimax estimator.

THEOREM 4. Suppose that the assumptions of Theorem 2 are fulfilled and $t(X)$ is an estimator such that $\sup _{\theta}(t, \theta)$ is equal to ICRB. Then $t$ is minimax.

Example 1. Let us consider Example 1 from Section 3. Since $t(X)$ $=(s+1)^{-1} X$ gives

$$
\sup _{\theta} R(t, \theta)=(s+1)^{-1}
$$

by Theorem 4 the estimator $t$ is a minimax estimator of $\tau(\theta)=\theta^{-1}$ for $\theta \in\left(0, \theta_{0}\right)$ or $\theta \in\left(\theta_{0}, \infty\right)$.

Example 2. Consider Example 2 from Section 3 and $t(X)=X$ as an estimator of $\tau(\theta)=\theta$. Since

$$
\sup _{\leftrightarrow} R(t, \theta)=1
$$

by Theorem 4 the estimator $t$ is a minimax estimator for $\Theta=\left(-\infty, \theta_{0}\right)$ of $\Theta=\left(\theta_{0}, \infty\right)$. Observe that $t$ is unbiased.

Although the Cramer-Rao inequality is a basis for ICRB, Theorem 4 may be applied also for not exponentially distributed estimators. Moreover, the next example shows that the extension of the underlying distributions cannot be reduced to the Joshi modification of the exponential family (see [3]).

We start with the following

Proposition. Let

$$
\begin{gathered}
\mathrm{E}_{\theta} X=m_{1} \tau(\theta), \quad \mathrm{E}_{\theta} X^{2}=m_{2} \tau^{2}(\theta)-m_{3} \tau(\theta), \\
\tau_{0}=\underset{\theta}{\inf \tau(\theta)>0 \quad \text { and } \quad m_{3} \geqslant-\tau_{0}\left(m_{2}-m_{1}^{2}\right)^{2} m_{1}^{-2} .}
\end{gathered}
$$

If $\Theta$ contains a sequence $\left\{\theta_{i}\right\}$ such that $\tau\left(\theta_{i}\right) \rightarrow x(i \rightarrow x)$ and

$$
\lim _{i \rightarrow \infty} \tau^{2}\left(\theta_{i}\right) h\left(\theta_{i}\right)=\sup _{\theta} \tau^{2}(\theta) h(\theta)=K,
$$


then $m_{1} / m_{2} X+b$, where

$$
\begin{aligned}
\tau_{0}\left[1-m_{1}^{2} / m_{2}-\right. & \left.\sqrt{\left(1-m_{1}^{2} / m_{2}\right)^{2}+m_{1}^{2} / m_{2}-m_{3} / \tau_{0}}\right] \\
& \leqslant b \leqslant \tau_{0}\left[1-m_{1}^{2} / m_{2}+\sqrt{\left(1-m_{1}^{2} / m_{2}\right)^{2}+m_{1}^{2} / m_{2}-m_{3} / \tau_{0}}\right],
\end{aligned}
$$

is a minimax estimator of $\tau(\theta)$ in the class $\mathscr{L}=\{a X+b ; a, b \in \boldsymbol{R}\}$ under the squared error loss with the weight function $h$, and the minimax value is equal to $K\left(1-m_{1}^{2} / m_{2}\right)$. Moreover, if additionally $b \geqslant 1-m_{1}^{2} / m_{2}$, then $m_{1} / m_{2} X+b$ is admissible in the class $\mathscr{L}$ as well. omitted.

Since the proof of the Proposition is simple but rather long, it is

Example 3. Suppose that the r.v. $X$ from Example 1 (a) of Section 3 is disturbed by a random error $U$, the distribution of which, in general, depends on $\theta$ and satisfies the regularity conditions (a)-(e). From the Proposition it follows that $t(X)=(s+1)^{-1} X+\theta_{0}^{-1}(s+1)^{-1}$ is a minimax and admissible estimator in the class of linear estimators, and the minimax value is equal to $(s+1)^{-1}$. A statistician observes $Y=X+U$ instead of $X$ and would use $t(Y)=(s+1)^{-1} Y+\theta_{0}^{-1}(s+1)^{-1}$ rather than $t(X)$. Assume, for simplicity, that $U$ and $Y$ are independent and $\mathrm{E}_{\theta} U \geqslant 0$. Clearly, $Y$ is not exponentially distributed in this case and

$$
\begin{aligned}
R(t(Y), \theta)= & R(t(X), \theta)+\theta^{2}(s+1)^{-2} \mathrm{E}_{\theta} U^{2}+ \\
& +2 \theta^{2} /(s+1)^{2}\left[1 / \theta_{0}-(s+2) / \theta\right] \mathrm{E}_{\theta} U .
\end{aligned}
$$

If (i) $\mathrm{E}_{\theta} U^{2} \leqslant \theta_{0}^{-2}$ and (ii) $I_{U}(\theta) \theta^{2} \rightarrow 0(\theta \rightarrow 0)$, where $I_{U}(\theta)$ is the Fisher information corresponding to $U$, then, by Theorem $4, t(Y)=(s+1)^{-1} Y$ $+\theta_{0}^{-1}(s+1)^{-1}$ is a minimax estimator of $\tau(\theta)=\theta^{-1}$. In fact, (i) implies $\sup _{\theta} R(t(Z), \theta)=(s+1)^{-1}$ and from (ii) it follows that ICRB for the statistic $Y$ is equal to $(s+1)^{-1}$.

The following result is implied by Theorem 3 and the definition of admissible estimators.

THEOREM 5. Suppose that the assumptions of Theorem 3 are fulfilled and $t(X)$ is an estimator such that

$$
R(t, \theta)=\tau^{2}(\theta) h(\theta)\left[\left(\tau^{\prime}(\theta) / \tau(\theta)\right)^{-2} I(\theta)+1\right]^{-1} .
$$

Then $t$ is admissible.

Example 4. Consider Example 1 from Section 3 for $\theta \in(0, \infty)$. It is easy to check that the right-hand side of $(31)$ is equal to $(s+1)^{-1}$. Since the risk of $t(X)=(s+1)^{-1} X$ is equal to $(s+1)^{-1}$, the estimator $t$ is an admissible (and minimax, of course) estimator of $\tau(\theta)=\theta^{-1}$.

Acknowledgement. The author wishes to thank Prof. W. Krysicki, Prof. D. Szynal and Prof. R. Zieliński for many valuable suggestions. 


\section{References}

[1] C. R. Blyth and D. N. Roberts, On inequalities of Cramer-Rao type and admissibility proofs. pp. 17-30 in: Proc. Sixth Berkeley Symp. Math. Statist. Prob. 1972.

[2] I. A. Ibragimov and R. Z. Has'minskiI, Statistical estimation, Asymptotic theory, Springer-Verlag, Berlin 1981.

[3] V. M. Joshi, On the attainment of the Cramer-Rao lower bound, Ann. Statist. 4 (1976), pp. 998-1003.

[4] S. Zacks. The therry of statistical inference, J. Wiley, New York 1971.

INSTITL'TE OF MATHEMATICS

TECHNICAL UNIVERSITY OF LÓDŹ

90-924 I.ÓDŹ

Received on 1984.10.04;

revised version on 1986.02 .20 